\title{
Menschenrechtsfilm oder Kriegsporno: Was legitimiert Gewaltdarstellungen?
}

Vanessa Rüegger*

Ist es legitim, in einem Spielfilm die Vergewaltigung einer Frau darzustellen, um das Publikum über Menschenrechtsverletzungen aufzuklären? Und fällt die Antwort auf die Frage anders aus, wenn dieselbe Vergewaltigungsszene zu einem späteren Zeitpunkt auf einer Pornoseite im Internet auftaucht? Die Frage nach der Rechtfertigung von Kriegs- und Gewaltdarstellungen ist mindestens so alt wie die frühen Kinofilme über den Ersten Weltkrieg und drängt sich auch anlässlich der jüngst veröffentlichten Bildern aus der Ukraine oder aus Syrien erneut auf. Die Suche nach einer Antwort führt jedoch zu einem Legitimationsparadox: Plausible Gründe für und gegen die Darstellung von Gewalt stehen einander gegenüber. Wie aber reagiert das Recht? Das Recht setzt sich mit einem repressiven Grundsatzentscheid über das Legitimationsparadox hinweg, um sich unter Anwendung einer Ausnahmeklausel dennoch in jedem Einzelfall die Möglichkeit offen zu halten, auch anders zu entscheiden. Dadurch behauptet das Recht seine Souveränität über die Kunst und bewahrt die Illusion seiner Einheit gegenüber der unkontrollierbaren Vielfalt des Lebens.

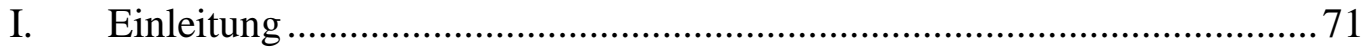

II. Krieg und Kunst - ein Legitimationsparadox ........................................ 72

III. Legitimation von Bildern sexueller Gewalt im Recht ............................ 74

1. Grundsatzentscheid: Bilder sexueller Gewalt sind verboten ............74

2. Ausnahmeklausel: Kunst legitimiert Bilder sexueller Gewalt .........76

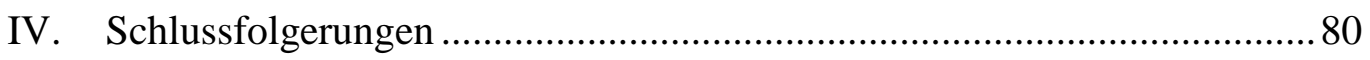

Zitiervorschlag: Vanessa Rüegger, Menschenrechtsfilm oder Kriegsporno:

Was legitimiert Gewaltdarstellungen?, in: sui-generis 2014, S. 70

URL: $\quad$ sui-generis.ch/6

DOI: $\quad$ https://doi.org/10.21257/sg.6

\footnotetext{
* Dr. iur., Assoziierte Professorin für öffentliches und internationales Recht, Universitäre Fernstudien Schweiz, und Habilitandin an der Universität Basel. Anstoss für die vorliegenden, skizzenhaften Ausführungen gaben die Diskussionen mit der Regisseurin Juanita Wilson, dem Fotografen und Filmemacher Nick Danziger und den Teilnehmern der 9. Summer School in Cinema, Human Rights and Advocacy an der National University of Ireland, Galway.
}

Dieses Werk ist lizenziert unter einer Creative Commons Namensnennung - Weitergabe unter gleichen Bedingungen 4.0 International Lizenz. 


\section{Einleitung}

1 Der berührende Film «As if I am not there» (2010) von Juanita Wilson (basierend auf dem gleichnamigen Roman von Slavenka Drakulić) thematisiert das Leben internierter Frauen in einem Gefangenenlager während dem Jugoslawienkrieg (1992-1995). Feinfühlig, nuanciert und in überzeugender Bildsprache erzählt die Regisseurin die Geschichte der jungen Samira, die im Lager - wie unzählige andere Frauen auch - wiederholt von Soldaten vergewaltigt wird. Die explizite Darstellung sexueller Gewalt beschränkt sich auf eine einzige Szene und zeigt die erste Vergewaltigung Samiras: Drei Soldaten mittleren Alters und von unvorteilhaftem, ungepflegtem Äusseren drängen die junge Frau in einen engen Raum. Einer der Soldaten reisst die Knöpfe ihrer blauen Bluse auf, zückt sein Messer und schlitzt den Büstenhalter an der engen Stelle zwischen den vollen Brüsten auf. Nackt steht Samira zitternd im Raum, versucht den Schambereich zu verdecken. Ein Soldat packt sie, wirft ihren Oberkörper auf einen alten Holztisch, drückt ihren Kopf auf die Tischplatte. Ein anderer öffnet seine Gurtschnalle und dringt von hinten lustvoll in den wehrlosen Körper der Frau ein. Beinahe in Echtzeit folgt nun die orgastische Befriedigung der drei Männer, während Samira als Ausweg nur die Flucht des Blicks an eine an der Wand klebenden Fliege bleibt.

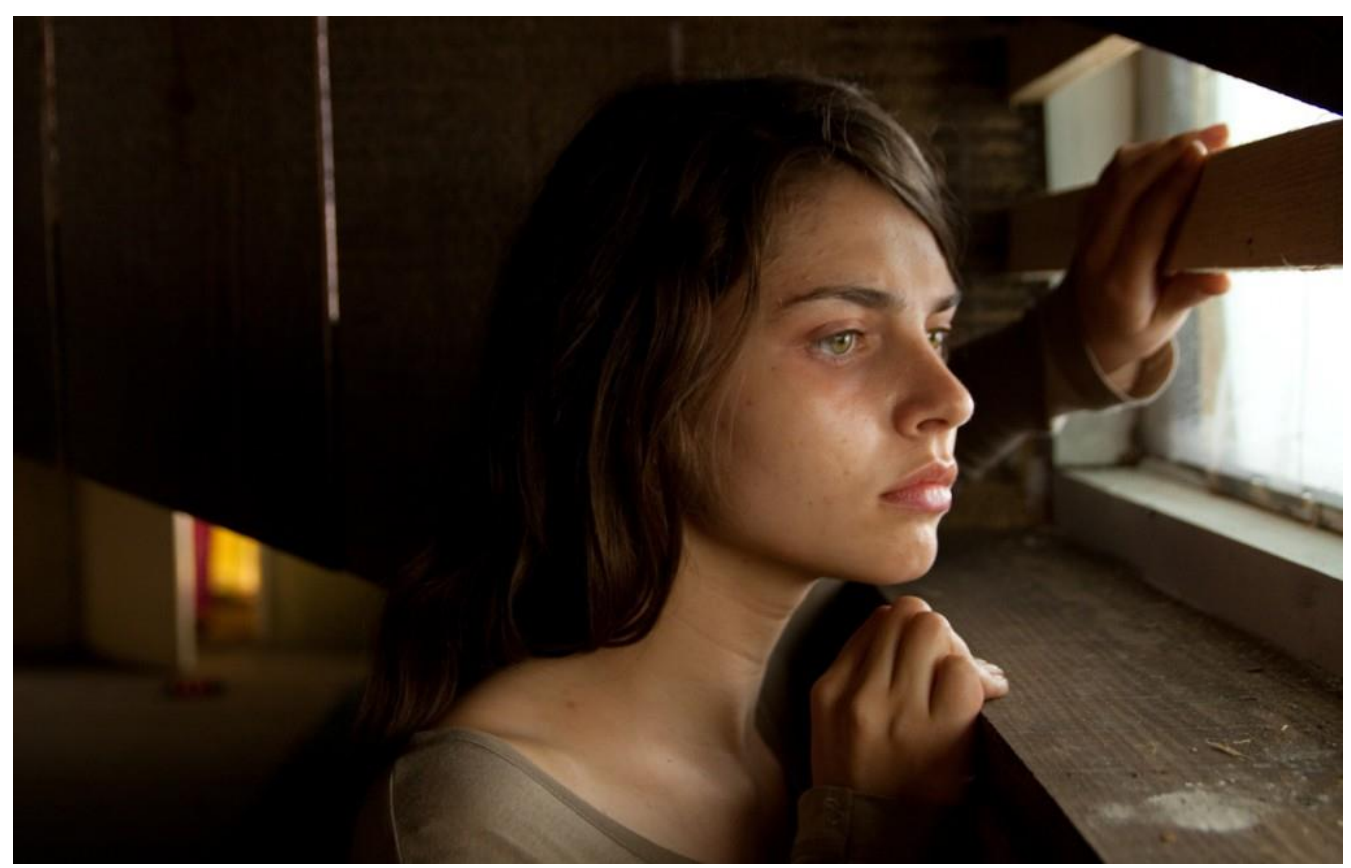

Video Still aus As if I am not there ${ }^{1}$

Obwohl die Szene mit Nacktaufnahmen sparsam umgeht und jegliche Bezüge zum Erotischen vermeidet, hat sie, nach eigenen Aussagen der Regisseurin, den Weg auf eine pornografische Internetseite gefunden. Während die
Sequenz also auf die einen Betrachter schockierend wirkt und nur schwer zu ertragen ist, unterstützt ihre Wahrnehmung unzweifelhaft die Befriedigung der sexuellen Bedürfnisse anderer. Dieselbe Szene erhält also eine

Mit bestem Dank für Auswahl und Publikationserlaubnis an Juanita Wilson. 
grundlegend andere Bedeutung je nach Betrachtungsweise. Nicht nur, aber auch deshalb, wirft die Vergewaltigungsszene des Films eine grundsätzliche Frage über die Darstellung von Krieg und Gewalt in der Kunst auf: Was legitimiert Bilder von schweren Menschenrechtsverletzungen, insbesondere von sexueller Gewalt, in einem Spielfilm oder in einer anderen Form bildlicher Darstellung? ${ }^{2}$

\section{Krieg und Kunst - ein}

\section{Legitimationsparadox}

Die Frage nach der Rechtfertigung von Kriegs- und Gewaltdarstellungen in der Kunst ist mindestens so alt wie die frühen Kinofilme über den Ersten Weltkrieg. Ausgehend vom englischen Film The Battle of the Somme und dessen deutscher Gegenüberstellung Bei unseren Helden an der Somme (beide Filme wurden 1916 veröffentlicht) fragt ein unbenannter Autor in der NZZ vom 25. Mai 1917 «welchem tiefern Zweck diese Vorführungen dienen sollen, selbst wenn es ihnen gelungen sein sollte, den Krieg so darzustellen, wie er wirklich aussieht, und im Zusammenfassen der einzelnen Geschehnisse eine Realität der Darstellung zu erreichen.» Für den Autor des Beitrags steht denn auch fest, dass die Darstellung des Kriegs im Kino nicht mit pazifistischen Gründen zu rechtfertigen ist. Die Darstellung kriegerischer Grausamkeiten vermag zwar Gefühle zu wecken. Diese verdichten sich jedoch nicht zwangsläufig zu intellektuellen Argumenten gegen den Krieg und damit zu deren Ablehnung: «Das, was man sieht, bleibt wesenslos, weil es uns nicht das vermitteln kann, was den wesentlichen Inhalt ausmacht.» Weil der Film als Reproduktion einzelner Kriegshandlungen erst dann zu öffentlicher Aufmerksamkeit gelangen kann, wenn diese bereits der Geschichte angehören, enthält er möge er noch so realistisch inszeniert sein ein fiktives Moment. Für den Autor ist es aber genau «jenes Moment spielerischer Bewegungen inmitten, oder nach bereits überstandener Gefahr - was unter Umständen sogar anziehend wirken kann, weil es nicht vernichtet und zerstört.» $^{3}$

4 Ähnlich argumentiert ein knappes Jahrhundert später auch Susan Sontag. In ihrem Essay Das Leiden anderer betrachten weist sie darauf hin, dass Fotografien, auch dokumentarische, nicht einfach die Abbildung der 'Realität' sind, sondern bereits eine gewisse Auswahl treffen und das Geschehene nie, auch nicht nur annäherungsweise darzustellen vermögen. Fotografien vom Leiden anderer werden von jedem Betrachter unterschiedlich wahrgenommen und führen zu unterschiedlichen Reaktionen. Kommunikationsinhalte, die einem Bild entnommen werden, entziehen sich den ursprünglichen Absichten der Fotografin. Kriegsbilder motivieren den Betrachter nicht notwendigerweise, gegen Gewalt zu denken oder handeln, sondern unterstützen unter Umständen auch Gewalthandlungen. Gewaltbilder befriedigen nach Sontag zudem immer auch eine voyeuristische Faszination und können trotz, oder gerade auf Grund des gewalttätigen Umfelds, in dem sie entstanden sind, sexuell konnotiert sein: «Alle Bilder, die die Verletzung eines anziehend wirkenden Körpers darstellen, sind bis zu einem gewissen Grade pornographisch.» ${ }^{4}$ Sontag selbst stellt
2 Die Begriffe Bilder und Darstellungen sind im vorliegenden Artikel im weiten Sinn zu verstehen und umfassen Fotografien, Zeichnungen, Malereien, Abbildungen und bewegte Bilder. Auch der Begriff Kunst ist im vorliegenden Artikel im weiten Sinn zu verstehen und umfasst sämtliche den Künsten zugeteilten Formen wie beispielsweise die Fotografie, die bildenden Künste, die neuen Künste, den Film und das Theater.

3 Abgedruckt im NZZ Fokus Nr. 56 (2014), 73 (Autor nicht benannt).

4 Sontag Susan, Das Leiden anderer betrachten, 5. Aufl., Frankfurt a. M. 2013, 111. 
sich auf den Standpunkt, dass Bilder über die Auswirkungen von schweren Gewalttätigkeiten uns in Erinnerung halten, welche Greueltaten Menschen im Stande sind anderen zuzufügen. Nach Sontag wäre es jedoch vielmehr unbedingt notwendig, sich über die Betrachtung des Leidens anderer hinaus Gedanken darüber zu machen, wie weitere Gewalt verhindert werden könnte. ${ }^{5}$

Zwei Argumente für die Darstellung von Krieg und Gewalt in sogenannten Menschenrechtsfilmen werden gemeinhin angebracht: Erstens sollen die Bilder dazu dienen, die Öffentlichkeit über die Geschehnisse zu informieren und aufzuklären. Zweitens sollen die Zuschauer durch Bilder für die Bedeutung der Menschenrechte und dem aus Menschenrechtsverletzungen zugefügten Leiden sensibilisiert werden, um sie zu aktiven Befürwortern der Menschenrechte auszubilden. Diesen Überlegungen stehen die Argumente gegenüber, wonach bei Filmen über Krieg und Gewalt auf eine explizite Darstellung zu verzichten ist, weil sich deren visuelle Reproduktion negativ auf den Betrachter auswirken könnte, sei es weil ihn die Bilder zu einem unerwünschten oder gar unrechtmässigen Tun motivieren könnten, oder weil ihn die Bilder psy- chisch belasten. Ebenfalls gegen die Visualisierung von Gewalt spricht das Argument, dass nicht nur der Akt der Gewaltausübung selber untolerierbare Machtverhältnisse generiert, sondern dessen Darstellung dieselben Machtverhältnisse mit jeder Betrachtung reproduziert und damit auch perpetuiert. ${ }^{6}$

6 Die Suche nach einer Antwort auf die Frage nach der Legitimität visueller Reproduktionen sexueller Gewalt führt also zu einem Paradox: Wird der Darstellung von Gewalt eine gewisse Wirkung zugeschrieben, dann kann deren Verwendung zwar durch den Aufklärungsgedanken gerechtfertigt werden. Zugleich bedeutet das aber auch, dass unerwünschte Einflüsse auf den Betrachter nicht ausgeschlossen werden können, weil die auf einem Bild erkennbaren Formen unterschiedliche Kommunikationsinhalte übermitteln. Dieselben Bilder kriegerischer Gewalt, die allenfalls zu Aufklärungszwecken legitimiert werden können, sind zugleich auch Kommunikationsträger unerwünschter Kommunikationsinhalte, indem sie beispielsweise zu weiteren Gewalthandlungen motivieren, sexuelle Bedürfnisse bedienen, oder für die abgebildeten Opfer oder ihre Familien ein schmerzhafter Anblick sind. Diese unerwünschten Kommunikationsin-

\footnotetext{
Sontag, Das Leiden anderer betrachten, $139 \mathrm{ff}$.

Bronkhorst Daan, The Human Rights Film, Reflection on its History, Principles and Practices, Amnesty International Film Festival, Amsterdam 2004; Tascon Sonia, Considering Human Rights Films, Representation, and Ethics: Whose Face? in: Human Rights Quarterly, 34/3 (2012) 864, 865 f.; Sontag, Das Leiden anderer betrachten, insbes. 25 ff., 71 ff. und 121 f.; Foster Hal, Obscene, Abject, Traumatic, in: Douzinas Costas/Nead Lynda (Hrsg.), Law and the Image, The Authority of Art and the Aesthetics of Law, Chicago/London 1999, 240 ff.; Kern Markus, Kommunikationsgrundrechte als Gefahrenvorgaben (Diss.), Freiburg 2012, 1 f. Siehe auch die aktuelle Diskussion über die Bilder der Opfer des in der Ukraine verunglückten Passagierflugzeugs MH17, beispielsweise im Tagesanzeiger vom 31. Juli 2014: Franklin Stuart, Wir brauchen mehr Beweise für das Böse - nicht weniger; und ebenda
}

am 2. August 2014: di Falco Daniel, Wirklichkeit, Welche Wirklichkeit?. Anlass zu ähnlichen Diskussionen bietet auch Bildverbreitung von Exekutionen durch islamistische Organisationen, wie beispielsweise jüngst die Bilder der Enthauptung eines amerikanischen Journalisten zeigen, siehe beispielsweise Tagesanzeiger vom 21. August 2014: Widmer Michèle, Warum darf Youtube dieses Video zeigen? Einen Überblick über die feministische Diskussion rund um das Legitimationsparadox in Bezug auf Pornographie und sexuelle Gewalt vermitteln Büchler Andrea/Cottier Michelle, Legal Gender Studies, Rechtliche Geschlechterstudien, Zürich/Baden-Baden 2012, 324 ff. Als Beispiel für einen nuancierten Zugang zum Thema Krieg und Kunst siehe Halasa Malu/Omareen Zaher/Mahfoud Nawara (Hrsg.), Syria speaks, Art and culture from the frontline, London 2014. 
halte unterlaufen also wiederum die Legitimität visueller Reproduktionen von Gewalt. Die Frage nach der Legitimität von Kriegs- und Gewaltbildern führt unter diesen Annahmen zu einem unauflösbaren Dilemma. Wird Bildern hingegen jegliche Wirkung abgesprochen, wäre ihre Reproduktion mit dem Aufklärungsgedanken nicht mehr zu rechtfertigen, die Gründe für deren Unterbindung wären jedoch gleichsam hinfällig geworden, das Paradox hätte sich in der Indifferenz aufgelöst. ${ }^{7} \mathrm{Ob}$ sich die Darstellung sexueller Gewalt wie beispielsweise in der Vergewaltigungsszene in As if I am not there moralisch rechtfertigen lässt ist folglich erstens davon abhängig, ob den Bildern zugemutet wird, sich auf ihre Betrachter auszuwirken, und zweitens, wie man das damit vorliegende Legitimationsparadox zu umgehen versucht.

\section{Legitimation von Bildern sexueller Gewalt im Recht}

Die Rechtfertigung von Gewaltdarstellungen führt, sofern den Bildern Wirkungskraft zugemutet wird, zu einer paradoxen Argumentationsstruktur, wonach plausible Gründe für und gegen die Darstellung von Gewalt einander gegenüberstehen. Wie aber geht das Recht mit dem Legitimationsparadox visueller Darstellungen von sexueller Gewalt um?

Das Recht muss Unentscheidbares entscheiden. Das bedeutet auch, dass das Recht jeden ihm vorgelegten Fall über die Rechtmässigkeit einer Gewaltdarstellung mit einem Urteil abschliessen und sich damit unweigerlich der einen oder anderen Seite des Paradoxes zuordnen muss. Wie also reagiert das Recht? Es findet einen Ausweg, um den Erwartungen an die Rechtssicherheit durch die Festlegung eines

Vertreter der 'Auflösungsstrategie' finden sich auch im rechtsdogmatischen Diskurs zum Thema, siehe unten
Grundsatzes $\mathrm{zu}$ genügen und sich dennoch vorzubehalten, auch anders entscheiden zu können. Das Recht verbietet Gewaltdarstellungen grundsätzlich und fingiert damit normative Konstanz. Um im Einzelfall dennoch variabel entscheiden zu können, führt das Recht zusätzlich eine Ausnahmeklausel ein und setzt den Richter als Wächter an dessen Tor. Das Recht setzt sich scheinbar mit einem repressiven Grundsatzentscheid des Gesetzgebers über das Legitimationsparadox hinweg, um sich aber unter Anwendung einer Ausnahmeklausel dennoch in jedem Einzelfall die Möglichkeit offen zu halten, anders zu entscheiden.

\section{Grundsatzentscheid: Bilder sexueller Gewalt sind verboten}

9 Ohne auf die rechtsdogmatischen Konstruktionen im Detail einzugehen, kann der gekoppelte 'Konstanz-Varianz-Mechanismus' - so wie er im schweizerischen Recht vorliegt - in groben Umrissen wie folgt skizziert werden: Das Recht unterscheidet zwischen dem Akt der Vergewaltigung und seiner visuellen Darstellung. Das Strafrecht sanktioniert sowohl den Akt der Vergewaltigung (Art. 190 StGB) als auch dessen visuelle Darstellung (Art. 135 StGB, respektive Art. 197 Abs. 4 f. StGB (aArt. 197 Ziff. 3 und 3 bis $\mathrm{StGB}^{8}$ )). Während die Vergewaltigung selbst mit Freiheitsstrafe von einem bis zu zehn Jahren bestraft wird, weist das Strafrecht der Darstellung der Vergewaltigung eine mildere Strafe zu (je nach Tatbestand Geldbusse oder Freiheitsstrafe bis zu drei Jahren). Deutlich zum Ausdruck kommt zudem die Wertung, dass das Recht gewissen Darstellungen 'tatsächliche' Vorgänge zuordnet, was wiederum zu einem erhöhten Strafmass führt (vgl. Art. 197 Abs. 4 f. StGB). Implizit folgt daraus, dass das Recht

$8 \quad$ BBl 20127571. 
davon ausgeht, das visuelle Darstellungen sexueller Gewalt nicht notwendigerweise tatsächlichen Gewalthandlungen entsprechen. ${ }^{9}$ Das Recht unterteilt also die Wahrnehmung sexueller Gewalt in die Kategorien Realität (der Akt der Vergewaltigung) und Fiktion (die visuelle Darstellung des Akts der Vergewaltigung), wonach gewisse visuelle Wahrnehmungen nicht der 'Realität' entsprechen, sondern vielmehr als 'Fiktionen' differenziert zu betrachten sind. Das Recht übernimmt damit die Unterscheidung zwischen realer und fiktionaler Realität des Kunstsystems und orientiert damit das Rechtssystem an der Vorstellung einer Realitätsverdoppelung. ${ }^{10}$

Weiter anerkennt das Recht, dass Bilder beim Betrachter etwas auslösen. Das Recht mutet dem Bild subversives Potential zu, welches autoritärer Kontrolle bedarf, um die Allgemeinheit und das Individuum vor potentiellen Gefahren zu schützen. Das hauptsächlich von Art. 135 StGB, respektive Art. 197 StGB, geschützte Rechtsgut ist nicht die körperliche Integrität der Dargestellten, sondern der Schutz des jugendlichen Betrachters vor schädigenden Einwirkungen auf seine Entwicklung, der
Schutz des erwachsenen Betrachters vor seinen gesellschaftlich unerwünschten Neigungen, wie auch die Prävention von Nachahmungstaten. ${ }^{11}$ Auch der damit avisierte Schutz der Menschenwürde bezieht sich nicht primär auf die Würde der Darsteller, sondern auf die Würde der Betrachter. Deren Menschenwürde wird durch Gewaltdarstellungen dadurch verletzt, «dass der Mensch als Bestie dargestellt und dem Betrachter (oder Zuhörer) zugemutet wird, an grausamer Quälerei Interesse oder gar Lust zu finden - eine im Gegensatz zur Pornographie gänzlich unmenschliche und jedenfalls höchstens den schwärzesten Schatten der Seele entspringende Lust. Der Tatbestand schützt gewissermassen die elementare Würde so empfindender Menschen gegen ihre eigenen perversen Triebe.» ${ }^{12}$ Das Recht befürchtet als Wirkung visueller Darstellungen beim Betrachter, dass sich diese «aufdringlich in der Phantasiewelt der Menschen einnisten», die «menschen- und wertfreundliche, gewaltfeindliche Geisteshaltung» abstumpfen, einen «verrohenden Einfluss» ausüben und «womöglich zu einer erhöhten Gewalttätigkeit» führen. ${ }^{13}$
9 BB1 2012 7571, 7620 f.; Weder, in: StGB-Kommentar, Art. 197 N 20; Heimgartner Stefan, Weiche Pornographie im Internet, AJP 2005 1482, 1483; Urteil Bezirksgericht Zürich vom 16. Januar 1992, SJZ 89/1992, 160, 162 E. 2.3.

10 Luhmann Niklas, Die Kunst der Gesellschaft, Frankfurt a. M. 1997, 229 ff.

11 Stratenwerth Günther/Wohlers Wolfgang, StGB-Handkommentar, 3. Aufl. 2013, Art. 135 StGB N 1; Donatsch Andreas, in: StGB-Kommentar, 19. Aufl. 2013, Art. 135 StGB N 1; Weder Ulrich, in: StGB-Kommentar, 13. Aufl. 2013, Art. 197 N 1, 8, 12; Trechsel Stefan, StGB-Praxiskommentar, 2008, Art. 135 StGB N 2 f. und Art. 197 StGB N 10; Heimgartner, Weiche Pornographie, 1483; SJZ 89/1992, 160, 162 E. 2.3; Urteil des Zürcher Obergerichts vom 6. September 1995, in: SJZ 93/1997, 69, 70 E. 2.1.; BGE 117 IV 283 E. 4. c.; Urteil des Bundesgerichts 6B_875/2008 vom 12. November 2008.

12 Trechsel, StGB-Praxiskommentar, Art. 135 StGB N 9; SJZ 89/1992, 160. Das Bundesgericht und ein Teil der
Lehre sprechen dem Tatbestand der harten Pornographie indirekt auch schützende Wirkung gegenüber den Darstellern zu, siehe BGE 128 IV 201, E. 1.4.5; BGE 124 IV 106 E 3.c.aa., gl. M. Stratenwerth/Wohlers, StGB-Handkommentar, Art. 197 StGB N 1; Weder, in: StGB-Kommentar, Art. 197 N 12; aus Donatsch, in: StGB-Kommentar, Art. 135 StGB N 5, lässt sich nicht folgern, ob die Würde des Betrachters oder diejenige der Darsteller bezeichnet ist. Nach Kern, Kommunikationsgrundrechte als Gefahrenvorgaben, 181, bezieht sich der Schutz der Menschenwürde in Art. 135 StGB auf einen abstrakten Rechtswert.

13 Trechsel, StGB-Praxiskommentar, Art. 135 StGB N 2 f. und Art. 197 StGB N 10; kritisch zum Schutzzweck hingegen Gerny Daniel, Zweckmässigkeit und Problematik eines Gewaltdarstellungsverbots im schweizerischen Strafrecht (Diss.), Basel 1994; Riklin Franz, Sinn und Problematik einer «Brutalonorm» im Strafgesetzbuch, in: Das Menschenbild im Recht, Freiburg 1990, 405; Hagenstein Nadine, O tempora, o mores! oder Keine Macht den Killerspielen, AJP 2010 1293, $1301 \mathrm{f}$. Ausführlich zum Schutzzweck von Art. 135 StGB auch 
11 Bilder bestehen nach dieser rechtlichen Normierung also nicht nur aus wahrnehmbaren Formen, sondern diese Formen vermögen auch Einfluss auf ihren Betrachter auszuüben. Zur Erklärung des Handlungen provozierenden Einflusses eines Bildes auf dessen Betrachter hat sich in den Kulturwissenschaften in Anlehnung an die Sprechakttheorie der Begriff des Blickakts entwickelt: Der Betrachter belebt das Bild durch seinen Blick und tritt damit mit dem betrachteten Bild in eine 'quasiintersubjektive Beziehung mit transformativem Potential'. Welche Art von Wirkung dadurch beim Betrachter ausgelöst wird, lässt sich auch nach der Blickakttheorie weder voraussehen noch kontrollieren. Dass eine entsprechende Wirkung jedoch beim Betrachter unter bestimmten Umständen eine Veränderung auszulösen vermag, wird demnach angenommen. ${ }^{14} \mathrm{Ob}$ die befürchteten Wirkungen des Bildes tatsächlich eintreten ist für das Recht jedoch ohne Bedeutung: Das subversive Potential des Bildes ist positivrechtlich als abstrakte Gefährdung definiert, womit vermieden wird, dass entsprechende Wirkungen tatsächlich nachgewiesen werden müssen. ${ }^{15}$

\section{Ausnahmeklausel: Kunst legitimiert Bilder sexueller Gewalt}

12 Ausschlaggebend für die Frage nach der Legitimation von Bildern sexueller Gewalt ist nicht der Grundsatzentscheid des Gesetzgebers gegen Gewaltdarstellungen, sondern, dass das Recht eine Ausnahmeklausel einführt: Das Recht unterscheidet zwischen Bildern, die sich in der Darstellung sexueller Gewalt erschöpfen (Art. 135 StGB, respektive Art. 197 Abs. 4 f. StGB), und solchen, die einem schutzwürdigen wissenschaftlichen oder kulturellen Wert entsprechen (Art. 135 StGB, respektive Art. 197 Abs. 9 StGB [aArt. 197 Ziff. $5 \mathrm{StGB}^{16}$ ]). Während erstere als unrechtmässig gelten, sind letztere vom Straftatbestand der Pornographie, respektive demjenigen der Gewaltdarstellung, ausgenommen. Gewaltdarstellungen kommt nach Rechtsprechung und Lehre dann ein wissenschaftlicher oder kultureller Wert zu, wenn sie dazu geeignet sind, die Folgen individueller oder kollektiver Gewalt exemplarisch zu illustrieren und das kritische Bewusstsein für deren Verwerflichkeit zu wecken oder zu schärfen. ${ }^{17}$
Junod Valérie, Interdiction des jeux vidéos violents: Moral Kombat? Medialex 2012, 3 ff. Zur Umsetzung von Art. 135 StGB oder Art. 197 StGB durch den Ausschluss entsprechender Filmprojekte von der Filmförderung siehe Zufferey Nathalie/Aubry Patrice, Commentaire LCin, 2006, Art. 16 N 49 ff., 69 ff.

14 Fischer-Lichte Erika, Performativität, Eine Einführung, 2. Aufl., Bielefeld 2013, 147 ff.; differenziert zur Unterscheidung zwischen Wahrnehmung (psychisches System) und Kommunikation (soziales System) und der Konstruktion ihrer strukturellen Kopplung als Erklärung dafür, dass Bilder unterschiedlichen Interpretationen zugänglich sind, siehe Luhmann, Kunst, $20 \mathrm{ff}$.

15 BGE 133 II 136, E. 6.5.2; BGE 128 IV 201, E. 1.4.2; BGE 124 IV 106, E. 3.c. aa.; BBl 1985 II 1009, 1045 ff. Ausführlich zur empirischen Forschung über die Wirkungen von Gewaltdarstellungen Kern, Kommunikationsgrundrechte als Gefahrenvorgaben, 12 ff., insbes. 29 ff., welcher davon ausgeht, dass Gewaltdarstellungen negative Auswirkungen haben und sich deshalb deren rechtliche Einschränkung im Grundsatz rechtfer- tigen lässt; nach Hagenstein, Keine Macht den Killerspielen, 1297 f., ist die negative Wirkung von Gewaltdarstellungen zumindest nicht eindeutig auszuschliessen. Nach Stratenwerth/Wohlers, StGB-Handkommentar, Art. 135 StGB N 1, handelt es sich bei Art. 135 StGB wegen dem nicht nachweisbaren Nachahmungseffekt vielmehr um ein Verhaltensdelikt, mit welchem die Gesellschaft zum Ausdruck bringen will, dass derartiges Verhalten zu ächten ist. Kritisch zum Nachweis negativer Auswirkungen von 'brutalen Videospielen' auch Junod, Interdiction des jeux vidéos violents, $3 \mathrm{ff}$; ebenso Cornu Daniel, La violence dans les médias et ses limites, Medialex 2005, 92, 93 ff. Zur Negation der Wirkungskraft von Bildern als 'Auflösungsstrategie' des Legitimationsparadox auch oben, Fn. 7.

16 BB1 2012 7571, 7622.

17 BB1 1985 II 1009, 1045 ff.; BGE 131 IV 64, E. 10.4.; SJZ 89/1992, 160, 161 E. 2.1.; SJZ 93/1997, 69, 72 E. 3.1. und E. 3.2.; Stratenwerth/Wohlers, StGB-Handkommentar, Art. 135 StGB N 2; Trechsel, StGB-Praxiskommentar, Art. 135 StGB N 11, und Art. 197 StGB 
Adoption der Ausnahmeklausel für Bilder mit wissenschaftlichem oder kulturellem Wert führt dazu, dass das Rechtssystem Bilder, die das Kunstsystem nach dem systeminternen Code als Kunstwerke anerkennt, privilegiert behandelt und damit auch die Wertungen des Kunstsystems als rechtmässig qualifiziert. ${ }^{18}$ Für die Abgrenzung rechtmässiger von unrechtmässigen Bildern stützt sich das Rechtssystem folglich auf die im Kunstsystem entwickelten Kriterien. Damit kommt das Recht den Anforderungen aus den grundrechtlich geschützten Kommunikationsfreiheiten nach und würdigt zugleich die Verfassungsmässigkeit möglicher strafrechtlicher Einschränkungen. ${ }^{19}$

Massstab zur Beurteilung, ob der Darstellung wissenschaftlicher oder kultureller Wert zukommt, ist nicht der Durchschnittsbürger, sondern der künstlerisch aufgeschlossene Betrachter: «Wann einem Werk kultureller Wert im Sinne dieser Bestimmung beizumessen ist, lässt sich nicht in allgemeingültiger Form festlegen. Es liegt im Wesen der Kunst, dass sie ständig neue Formen annimmt, Normen sprengt und das Bestehende in Frage stellt, weshalb es nicht möglich ist, sie abschliessend zu definieren. Der Richter hat daher von Fall zu Fall über den kulturellen Wert eines Werkes zu entscheiden. Massgebend kann dabei weder das Selbstverständnis des Kunstschaffenden sein, noch - wie nach früherer Rechtsprechung zu Art. 204 aStGB - das Kunstverständnis des Durchschnittsmenschen. Da der Gesetzgeber mit dem revidierten Sexualstrafrecht nicht mehr den Schutz der Sexualmoral der Allgemeinheit bezweckte, sondern darum bemüht war, den Schutz auf klar umrissene Rechtsgüter des Einzelnen zurückzuführen, ist das Werk mit Blick auf den grundrechtlichen Schutz der Kunstfreiheit (Art. 21 BV; Art. 10 Abs. 1 EMRK; Art. 19 Abs. 2 UNO-Pakt II) vielmehr aus der Sicht eines künstlerisch aufgeschlossenen Betrachters zu beurteilen. Dies wird dem Richter in der Regel möglich sein, ohne einen Sachverständigen beizuziehen.» ${ }^{20}$ Damit grenzt sich die Formulierung bemerkenswert von der Rechtsprechung zu Art. 204 aStGB ab, welche zur Einschätzung des geschützten Sittlichkeitsgefühls in geschlechtlichen Dingen den 'normalen Menschen', respektive den 'normal empfindenden Betrachter' oder den 'normal empfindenden Bürger' als Massstab nahm. ${ }^{21}$

Aus den Ausführungen des Bundesgerichts im
N 19; bezüglich der Legitimation von Gewaltdarstellung in Informationssendungen siehe die analoge Argumentation im Entscheid der unabhängigen Beschwerdeinstanz für Radio und Fernsehen vom 5. Dezember 2003, Medialex 200461.

18 Eine Auseinandersetzung mit weiteren Bedeutungsgehalten der Worte 'wissenschaftlich' und 'kulturell' ist nicht Gegenstand des vorliegenden Beitrags.

19 Die Kommunikationsfreiheit steht damit quasi als freiheitlicher Gegenpol der hier diskutierten strafrechtlichen Restriktion gegenüber. Die rechtsdogmatische Struktur der Kommunikationsfreiheiten ist jedoch explizit nicht Gegenstand des vorliegenden Beitrags, siehe für ausführliche Hinweise auf die Fachliteratur aber Kern, Kommunikationsgrundrechte als Gefahrenvorgaben, insbes. 167 ff.; ebenso Peduzzi Roberto, Meinungs- und Medienfreiheit in der Schweiz (Diss.), Zürich/Basel/Genf 2004.

20 BGE 131 IV 64, E. 10.1.3; ausführlich auch SJZ
93/1997, 69, 71 f. E 3.1.: «Der dem Richter zugewiesene Entscheid lässt sich treffen, ohne dass es eines besonderen Kunstsachverstandes bedürfte, weshalb in der Regel auch kein Experte heranzuziehen ist; namentlich die Frage der Schutzwürdigkeit ist rechtlicher Natur und muss vom Richter selbst beantwortet werden. Mit der Verteidigung darf allerdings bezüglich dieser Frage gefordert werden, dass nicht einfach auf das Kunstverständnis eines Durchschnittsmenschen abgestellt wird, sondern der Massstab eines künstlerisch aufgeschlossenen Betrachters heranzuziehen ist. Dieser Anforderung hat der Richter, dem der Gesetzgeber einen entsprechenden Ermessensentscheid übertragen hat, zu genügen.» Trechsel, StGB-Praxiskommentar, Art. 135 N 11 und Art. 197 N 19; Weder, in: StGB-Kommentar, Art. 197 StGB N 29.

21 Siehe beispielsweise BGE 83 IV 19, E. 6; BGE 86 IV 19, E. 1; BGE 117 IV 457, E. 2.a. 
genannten Entscheid kann umgekehrt gefolgert werden, dass das Recht davon ausgeht, dass sich das unerwünschte subversive Potential von Gewaltdarstellungen stärker auf bestimmte Kategorien von Individuen auswirkt. Explizit findet sich diese Wertung im 'Blutgeil'-Urteil des Zürcher Obergerichts: «Eine Filmaufführung, welche den Anforderungskriterien eines Kunstsachverständigen allenfalls zu genügen vermag, kann durchaus beim Betrachter die Bereitschaft erhöhen, selbst gewalttätig zu agieren oder doch die Gewalttätigkeit anderer gleichgültig hinzunehmen. Diese Wirkung ist nicht zuletzt gerade bei demjenigen denkbar, der selbst über kein Kunstverständnis verfügt und deshalb eine allfällige - subtil ausgedrückte - Botschaft des Kunstschaffenden missdeutet oder nicht versteht. Bei der Beurteilung des schutzwürdigen kulturellen oder wissenschaftlichen Wertes ist daher auch dieser Aspekt vor Augen zu halten.» ${ }^{22}$

Damit nimmt das Recht zwar eine Erkenntnis aus der Kunsttheorie auf, wonach in der Betrachtung von Bildern keine Homogenität in der Interpretation vorausgesetzt werden kann. ${ }^{23}$ Das Recht koppelt diese Überlegung jedoch mit einer hierarchischen Klassifizie- rung der potentiellen Bildbetrachter: Die Gefahr der Auswirkungen visueller oder literarischer Darstellungen sexueller Gewalt besteht nach impliziten und expliziten Annahmen im Recht beim durchschnittlichen, nicht aber beim spezifisch ausgebildeten Betrachter. Besondere gedankliche Anstrengung verschafft dem Beobachter zudem Distanz zur Materie, womit auch der Ausschluss von Schriften aus dem Tatbestand von Art. 135 StGB auf Grund der gedanklichen Anstrengung des Lesens gerechtfertigt wird. ${ }^{24}$ Aufklärung in der Form von kultureller oder wissenschaftlicher Bildung vermag also einen Betrachter vor den befürchteten Auswirkungen zu schützen. Die Beobachtung des visuellen Akts der Vergewaltigung ist auch deshalb dann rechtmässig, wenn er höheren Zwecken als lediglich der menschlichen Triebbefriedigung dient.

17 Bemerkenswert ist schlussendlich die Präzisierung der Rechtsprechung, wonach die Kompetenz zur Grenzziehung zwischen unterschiedlichen Beobachtungsweisen der zur Frage stehenden Formen nicht etwa, wie auf Grund der Forderung nach einem 'künstlerisch aufgeschlossenen Betrachter' zu vermuten wäre, einem zumindest ansatzweise fachkundigen Beobachter zufällt, sondern dem Richter zugewiesen wird: ${ }^{25}$ Die Abgrenzung
E. 3.1

Für eine Übersicht über unterschiedliche Theorien über die Wahrnehmung visueller Darstellungen siehe Bertram Georg W., Kunst, Eine philosophische Einführung, Stuttgart 2011, 112 ff. und 170 ff.; und spezifisch mit Bezug auf die Kommunikationsfreiheiten Kern, Kommunikationsgrundrechte als Gefahrenvorgaben, 71 ff.; zur Heterogenität der Bilddeutungen auch Vismann Cornelia, Bildregime des Rechts - Rechtsregime des Bildes, in: Joly Jean-Baptiste/Vismann Cornelia/Weitin Thomas (Hrsg.), Bildregime des Rechts, Stuttgart 2007, 15 ff. Für eine systemtheoretische Deutung der Vorgänge siehe Luhmann, Kunst, $13 \mathrm{ff}$.

24 Stratenwerth/Wohlers, StGB-Handkommentar, Art. 135 StGB N 2; Trechsel, StGB-Praxiskommentar, Art. 135 StGB N 9; Donatsch, in: StGB-Kommentar, Art. 135 StGB N 1; ebenso BGE 133 II 136, E. 6.4: «Das
Bild ist konkret, wirkt emotional unmittelbarer, ganzheitlicher und unentrinnbarer als das Wort.» Das Zürcher Bezirksgericht hat zudem Abbildungen privilegiert gegenüber bewegten audiovisuellen Bildern beurteilt, mit der Begründung, dass die Fotografien des Films Blutgeil (SJZ 93/1997, 69) von ihrer Intensität her nicht mit einem Film vergleichbar sind, der durch Grossaufnahmen, Wiederholungen und Ton auf den Betrachter viel suggestiver wirkt, Flückiger Silvan, Strafbare «Blutgeil»-Filmbilder im Internet, Urteil des Bezirksgerichts Zürich vom 6. November 2002, Medialex 2003, 179 f. Nach den angegebenen StGB-Kommentaren fallen Schriften jedoch unter den Tatbestand von Art. 197 StGB; siehe zur Frage der Schriftlichkeit unter Art. 204 aStGB zudem ausführlich BGE 83 IV 19.

25 BGE 131 IV 64, E. 10.1.3, SJZ 93/1997, 69, 71 f. E 3.1. 
zwischen legitimen, weil rational kontrollierten und also juristisch geschützten Beobachtungen gegenüber illegitimen, weil rational unkontrollierten und also juristisch nicht schützenswerten Bildbetrachtungsweisen fällt einem Juristen zu. Während der Richter diese Position im jüngeren Urteil in BGE 133 IV 16 nach der zitierten Stelle zu folgern selbstbewusst einnimmt, ist im Blutgeil-Urteil noch eine gewisse Unsicherheit diesbezüglich erkennbar: «Dem Richter wird die heikle Aufgabe überbunden, im Einzelfall zu befinden, was von kulturellem und überdies zu schützendem Wert ist. [...] Und wenn im weiteren eingewendet wird, der Richter sei mangels ausreichender kulturgeschichtlicher Bildung regelmässig nicht in der Lage, etwa über den Wert ungewöhnlicher (z.B. avantgardistischer) Ausdrucksformen zu entscheiden, so ist dem entgegenzuhalten, dass es nicht darum geht, isoliert ein Kunstwerk als solches zu beurteilen. Die dem Richter zugewiesene Aufgabe besteht darin, sich im Sinne einer Interessenabwägung darüber auszulassen, ob eine grundsätzlich als schädlich befundene öffentliche Darstellung brutaler Handlungen im höheren Interesse kulturellen Schaffens hinzunehmen sei.» ${ }^{26}$

Das Recht geht also davon aus, dass der Jurist in der Form des Richters oder des Gerichtsschreibers gegenüber moralisch verwerflichen Betrachtungsweisen von Gewaltdarstellungen immun ist. Damit wird dem Juristen ohne weiteres zugemutet, dass er gegenüber sexuellen Darstellungen einen distanzierten Zugang be- wahrt und davon, entgegen anderen Individuen, die vor solchen Darstellungen durch präventive Zensur zu schützen sind, unberührt bleibt. Der Richter ist vor sich selbst nicht zu schützen, vielmehr wird er vom Recht zum Schutze anderer als Wächter an das Eingangstor zur Ausnahmeklausel gesetzt. ${ }^{27}$ Bedeutend ist, dass sich das Recht mit dieser eigenmächtigen Kompetenzzuweisung die Zäsur über die Grenze zwischen Recht und Unrecht selbst vorbehält. Das Recht gewinnt damit die eingangs der Kunst abgegebene Kompetenz über die Abgrenzung zwischen verwerflicher und schützenswerter, weil künstlerisch wertvoller Visualisierung zurück. Zwar anerkennt das Recht, dass die Kunst einer differenzierten Behandlung bedarf, ja eine solche verfassungsrechtlich geboten ist, und die Beurteilungskriterien des Kunstsystems von denjenigen des Rechtssystems abweichen. Mit der Figur der 'Schutzwürdigkeit' als Ermessensfrage, welche in einer Interessenabwägung vom Richter zu entscheiden ist, überführt das Rechtssystems den anfänglich der Kunst zugestandenen Freiraum jedoch souverän in den Kontrollbereich des Rechts zurück.

19 Durch die Einsetzung des Richters als souveräner Wächter über die Beurteilung der Schützenswürdigkeit von Gewaltdarstellungen versucht das Recht seine Unabhängigkeit gegenüber der Kunst zu gewährleisten und zugleich die unüberschaubare Vielfalt an der Schnittstelle zwischen möglichen Fallkonstellationen einerseits und visuellen Beobachtungsweisen andererseits $\mathrm{zu}$ kontrollieren. ${ }^{28}$ Letztendlich handelt es sich also auch um den Versuch, der

Authority of Art and the Aesthetics of Law, Chicago/London 1999, 203 ff. Kritisch zur Rolle des Richters auch Hagenstein, Keine Macht den Killerspielen, 1303.

28 Zum Bildregime des Rechts, verstanden als explizite und implizite Einwirkungen des Rechts auf die Bildgestaltung, auch Vismann, Bildregime des Rechts, 15 ff., 22: «Auch wenn sich im Rückblick auf ein intaktes 
Utopie der Einheit und Universalität des Rechts einen Schritt näher zu kommen. ${ }^{29}$

\section{Schlussfolgerungen}

20 Die Rechtfertigung von Gewaltdarstellungen unterliegt einer paradoxen Argumentationsstruktur, wonach plausible Gründe für und gegen die Darstellung von Gewalt einander gegenüberstehen. Die als Beispiel angeführte Vergewaltigungsszene aus dem Film As if I am not there wirkt auf die einen Betrachter schockierend, ihre Darstellung wird aber als notwendig zur Aufklärung über die Greuel des Jugoslawienkriegs erachtet. Zugleich wird die Szene von anderen unzweifelhaft für die Befriedigung sexueller Bedürfnisse verwendet, was als Argument gegen die Legitimität von Bildern sexueller Gewalt angeführt wird.

21 Wie ein Richter urteilen würde, hätte er über die Vergewaltigungsszene in As if I am not there nach Schweizerischen Recht zu entscheiden, bleibt auf Grund des 'Konstanz-Varianz-Mechanismus' eine nur mit Vermutungen zu beantwortende Frage: Ohne sich auf eine detaillierte strafrechtliche Argumentation einzulassen, deuten die Umstände darauf hin, dass die genannte Stelle im Film nach Art. 135 StGB zwar als Gewaltdarstellung klassifiziert werden könnte. Auch wäre es vorstellbar, dass die Gewaltszene, weil sie in einen differenzierten Gesamtkontext eingebettet ist, unter

Bildregime des Rechts dieses von seiner positiven Seite zeigt, als im eigentlichen Sinn bildgebendes Regime, bleibt die Frage, warum das Recht sich überhaupt der Bilder bemächtigt. Ein Verdacht liegt nahe: Bilder sind mehrdeutig und sie schüren Affekte. Beides ist für das Recht zwar nicht prinzipiell unerträglich, doch fordert es das Recht zu einer Reaktion heraus. Eindeutigkeit und affektfreie Rationalität herzustellen, gehört schliesslich zu seinen Grundoperationen.»

29 Zur Utopie 'Einheit des Rechts' ausführlich Kiesow Rainer Maria, L'unité du droit, 2014. Ausführlich zum einheitlich-regulativen Anspruch des Rechts über das die in Art. 135 StGB vorgesehene Ausnahmeklausel für Gegenstände mit schutzwürdigem kulturellem oder wissenschaftlichem Wert fällt. Weniger wahrscheinlich erscheint, dass der Film in einer Gesamtbetrachtung den Tatbestand der harten Pornographie nach Art. 197 Abs. 4 f. StGB zu erfüllen vermag. Offen bleibt hingegen, ob die von ihrem Kontext herausgelösten Bilder der Vergewaltigungsszene allenfalls unter den Tatbestand der harten Pornographie subsumiert werden könnten. Die Urheber einer derartig verfremdeten Darstellung der Bilder dürften sich kaum auf die Ausnahmeklausel von Art. 197 Abs. 9 StGB stützen können. ${ }^{30}$ Die Formulierungen über die Subsumtion verbleiben im Konjunktiv, denn: Was im vorgelegten Beispielfall Recht und Unrecht ist, kann nur ein richterliches Urteil abschliessend entscheiden.

22 Das Recht umgeht das Legitimierungsparadox visueller Darstellungen sexueller Gewalt unter Anwendung eines gekoppelten Mechanismus, dank dem das Recht den Erwartungen an die Rechtssicherheit genügt und sich dennoch vorbehält, auch anders entscheiden zu können: Das Recht setzt sich mit einem repressiven Grundsatzentscheid des Gesetzgebers über das Legitimationsparadox hinweg, um sich zugleich unter Anwendung einer Ausnahmeklausel dennoch in jedem Einzelfall die Möglichkeit offen zu halten, anders zu entscheiden. Das Recht normiert also Bilder sexueller Gewalt unterschiedlich, je nachdem ob

Bild auch Douzinas Costas/Nead Linda, Introduction, in: Douzinas Costas/Nead Lynda (Hrsg.), Law and the Image, The Authority of Art and the Aesthetics of Law, Chicago/London 1999, $1 \mathrm{ff}$.

30 Siehe beispielsweise die Klassifikation der 'Vergewaltigung einer Frau durch ihren Ehemann in der Küche' als harte Pornographie nach Art. 204 aStGB, wobei die Szene nicht in einem Spielfilm, sondern in einem als weiche Pornographie bezeichneten Film eingebettet war, BGE 117 IV 283 E 4. c.; Stratenwerth/Wohlers, StGB-Handkommentar, Art. 197 StGB N 5. 
sich die Darstellung auf Gewalthandlungen alleine beschränkt und damit - nach Annahmen des Rechts - einer (irrationalen) Triebbefriedigung dient, oder ob die Bilder in einen Kontext eingebettet sind, welcher nach Annahmen des Gesetzgebers eine rational-reflektierte Interpretation der Bilder ermöglichen soll. Das Recht räumt mit der Ausnahmeklausel der Kunst einen gewissen Freiraum im Umgang mit Bildmaterial ein. Indem es aber zugleich den Richter als Wächter vor das Eingangstor zur Freiheit setzt, behauptet das Recht seine Souveränität über die Kunst und bewahrt die Illusion seiner Einheit gegenüber einer unkontrollierbaren Vielfalt. 